\title{
The usefulness of ${ }^{99} \mathrm{~m}$ Tc-SestaMIBI scan in the diagnostic evaluation of thyroid nodules with oncocytic cytology
}

\author{
F Boi, M L Lai ${ }^{2}$, C Deias, M Piga ${ }^{1}$, A Serra ${ }^{1}$, A Uccheddu ${ }^{3}$, G Faa $^{2}$ and S Mariotti \\ Endocrinology and ${ }^{1}$ Nuclear Medicine, Department of Medical Sciences 'M. Aresu', ${ }^{2}$ Department of Cytomorphology and ${ }^{3}$ Department of Surgery and \\ Imaging, University of Cagliari, Cagliari, Italy
}

(Correspondence should be addressed to S Mariotti, Endocrinology, Department of Medical Sciences, Presidio di Monserrato, University of Cagliari, Strada Statale 554-bivio Sestu, I-09042 Monserrato, Cagliari, Italy; Email: mariotti@pacs.unica.it)

\begin{abstract}
Objective: To assess the relevance of ${ }^{99} \mathrm{~m}_{\mathrm{Tc}}$-SestaMIBI (MIBI) scan in the diagnostic evaluation of thyroid nodules with oncocytic cytology.

Subjects and methods: Twenty-four patients with a single (or prevalent) 'cold' solid nodule with Hürthle cells (HC) at fine needle aspiration cytology (FNAC) were studied. Cytological diagnosis of oncocytic metaplasia $(\mathrm{OM})$ or $\mathrm{HC}$ tumor $(\mathrm{HCT})$ was made when $\mathrm{HC}$ on the smear were comprised $10-75 \%$, or $>75 \%$. Nodules concentrating MIBI at early and late ( $2 \mathrm{~h}$ after washout) stages were considered MIBI-positive. In all cases histological findings were obtained after total thyroidectomy.

Results: FNAC was malignant or suspect for malignancy in 16 cases (six HCT and $10 \mathrm{OM}$ ) and not suspect in eight (two HCT and six OM). Histological examination revealed 14 malignant tumors (11 HCT and three OM), and 10 benign thyroid lesions (three HCT and seven OM). Sensitivity of FNAC for malignancy was $92.8 \%$ and specificity was $70.0 \%$; HCT were identified by FNAC in only $35.7 \%$ and $\mathrm{OM}$ in $70.0 \%$ of cases. No significant difference in MIBI positivity was found between malignant and benign thyroid nodules. The highest percentage of MIBI positivity was found in HCT (78.5\%), but MIBI-positive nodules were also observed in thyroid lesions with HC metaplasia (40.0\%). Conclusions: MIBI scintiscan has no value in differentiating malignant from benign HC thyroid neoplasias. Most HCT are MIBI-positive, but this scan is not sufficiently specific to differentiate true HC neoplasias from other thyroid lesions showing $\mathrm{HC}$ at FNAC, although an MIBI-negative scan strongly supports the absence of true HCT.
\end{abstract}

European Journal of Endocrinology 149 493-498

\section{Introduction}

Hürthle cells (HC) or oncocytes are polygonal thyroid follicular cells with abundant granular eosinophilic cytoplasm, containing numerous mitochondria $(1,2)$. The term 'oncocytic tumors' or HC tumors (HCT) is reserved for thyroid neoplasms composed exclusively or predominantly (over 75\%) of follicular cells exhibiting oncocytic features (3-5). The term oncocytic metaplasia $(\mathrm{OM})$ indicates the presence of a variable $(<75 \%)$ number of $\mathrm{HC}$, often mixed with follicular monomorphic and intermediate cells, in other benign or malignant thyroid lesions. OM is a non-neoplastic hyperplasia of $\mathrm{HC}$ and appears to be particularly frequent in Hashimoto's thyroiditis and nontoxic goiter (3-6). Classically, HCT are considered a variant of follicular neoplasms and may be classified as carcinomas or adenomas, based primarily on the architectural features of capsular or vascular invasion $(2-4$, 7). More recently, an $\mathrm{HC}$ variant of papillary thyroid carcinoma (HC-PTC) has also been recognized $(8,9)$.
Fine-needle aspiration cytology (FNAC) is considered the best procedure in the diagnostic evaluation of thyroid nodules (10), since in most cases it allows one to define the precise histotype in a simple and non-invasive fashion $(10,11)$. With particular regard to oncocytic nodules, FNAC does not appear to be a very reliable technique to distinguish $\mathrm{HC}$ adenomas from carcinomas $(1,4,5)$. As observed with other histotypes, thyroid ultrasound (12) may help to identify thyroid nodules with a high risk of malignancy, while conventional ${ }^{99} \mathrm{~m}$ Tc-pertechnetate $\left({ }^{99 \mathrm{~m}_{\mathrm{TcO}}}\right)$ thyroid scintiscan has presently only an ancillary role in distinguishing malignant from benign lesions (13). During the last decade, ${ }^{99} \mathrm{~m}$ Tc-SestaMIBI (MIBI), a lipophilic cationic molecule, was introduced as a myocardial perfusion and viability imaging agent (14-17). MIBI has been reported to accumulate in benign and malignant lesions, such as lung, brain, parathyroid tumors and bone lesions (18-21). Recently, a positive MIBI scan has been reported in different thyroid tumors $(22-27)$, but, when applied to unselected 
thyroid nodules, this procedure is not able to differentiate benign from malignant lesions $(24,27)$. Interestingly, however, MIBI scan has been found to be more frequently positive in HCT when compared with other thyroid neoplasias (27); however the relevance of this observation in the diagnostic evaluation of thyroid nodules is unclear, due to the very limited number of cases reported.

The aim of the present study was to evaluate the role of combined MIBI scintiscan and FNAC in the diagnostic evaluation of thyroid nodules with oxyphilic cytology, with particular regard to the differentiation of $\mathrm{HCT}$ from $\mathrm{OM}$ and the correct identification of malignant nodules.

\section{Materials, methods and patients}

\section{Patients}

A total of 24 euthyroid patients (20 women, age range 23-70 years, and four men, age range 42-72 years) with a single (or prevalent) thyroid nodule with $\mathrm{HC}$ at FNAC were included in this study. All patients were submitted to total thyroidectomy on the basis of clinical, echographic and/or cytological criteria independent from MIBI scan (see below). A summary of the main features of these patients, together with the final histology is reported in Table 1.

\section{Conventional and color flow Doppler sonography}

Thyroid ultrasonography (US) and color flow Doppler sonography (CFDS) using an Acuson 128 XP 10 color doppler system (Acuson Co., Mountain View, CA, USA) with a $7.5 \mathrm{mHz}$ linear electronic transducer, were performed. The examination included first a conventional gray scale US, followed by CFDS; all thyroid nodules were identified, localized, counted and their diameters measured. The images were obtained by transverse and longitudinal planes scanning. All nodules were solid iso-hypoecoic, often heterogeneous due to the presence of calcifications and fluid areas, with a maximum diameter ranging from 10 to $60 \mathrm{~mm}$. The CFDS patterns were classified (pattern $0-\mathrm{III})$ as previously reported (28). As shown in Table 1 , most of the patients presented intense intranodular vascularity, while the remaining nodules showed only peripheral blood flow.

\section{Thyroid function assays}

All hormonal and antibody assays were carried out by commercial kits. Serum free thyroxine (FT4) and free tri-iodothyronine (FT3) were assayed by RIA (Technogenetics, Milan, Italy); thyrotropin (TSH) by an ultrasensitive chemioluminescent assay (Ortho Clinical Diagnostic SpA, Milan, Italy); and anti-thyroperoxidase antibody (TPOAb) by RIA (Biocode, Liège, Belgium). Normal values were as follows: FT4, 8.4-20.4 pmol/l; FT3, 4.3-8.6 pmol/l; TSH, $0.2-3.0 \mathrm{mU} / \mathrm{l}$; antiTPOAb, <20 U/ml. All patients had normal thyroid function; as detailed in Table 1, 6/24 showed increased titers of TPOAb.

\section{Thyroid ${ }^{99 m}$ Tc-pertechnetate scintiscan}

Thyroid scintigraphy was performed by means of a computerized gamma-camera equipped with a pinhole collimator (Elscint, SP4; Haifa, Israel) $30 \mathrm{~min}$ after i.v. injection of $110 \mathrm{MBq}$ of ${ }^{99 \mathrm{~m}} \mathrm{TcO}_{4}$. Scans were performed in anterior, left-anterior oblique and right-anterior oblique projections. All patients showed a scintigraphic pattern characterized by a reduced uptake corresponding to a US position of prevalent thyroid nodules that often appeared typically 'cold'.

\section{Thyroid ${ }^{99 m}$ Tc-SestaMIBI scintiscan}

This was performed by means of the same gammacamera used for ${ }^{99 \mathrm{~m}} \mathrm{Tc}$ thyroid scan after i.v. injection of $740-1000 \mathrm{MBq}$ of MIBI without any specific preparation. Two images were acquired: an early image with an acquisition time of 5-10 min at $15 \mathrm{~min}$ and a late image with an acquisition time of $10-15 \mathrm{~min}$, $2-3 \mathrm{~h}$ after tracer administration. Visual inspection of the images was performed following the MIBI deposition in nodular and extranodular thyroidal tissue in both images. The nodules were described as 'hot' or MIBI-positive when the scintigraphic pattern showed increased localized uptake in both early and late images, and the extranodular tissue did not concentrate MIBI at a late stage.

\section{Fine needle aspiration cytology (FNAC) and histology}

US-guided FNAC was performed using 22-25-gauge needles and a $10 \mathrm{ml}$ syringe. The smears $(4-12$ for each nodule) were immediately fixed with Cytofix BioOptica, Milan, Italy and stained with hematoxylineosin (HE). In all cases a variable number (10-100\%) of follicular cells showed typical oncocytic changes. The criteria used for cytological evaluation were as follows: (a) For the evaluation of malignancy, monomorphic thyroid follicular cells with abundant colloid and without nuclear changes were considered non suspect. The presence of atypias such as nuclear enlargement, nuclear pleomorphism, binucleation and prominent nucleoli or cellular nests loosely cohesive with marked overlap, were considered suspect. A cytological diagnosis of PTC was made on the basis of classical features (papillae and/or characteristic nuclear changes such as grooves and pseudoinclusions). (b) With regard to differentiation between true HCT and OM, the cytology specimens were considered as suggestive of HCT when 
Table 1 Main clinical features of the study group.

\begin{tabular}{|c|c|c|c|c|c|c|c|c|c|}
\hline Patient no. & Age (years) & Sex & TPOAb & Nodule $(\mathrm{mm})$ US & CFDS* pattern & FNAC & Suspect for malignancy & MIBI scans & Histology \\
\hline 1 & 37 & $\mathrm{~F}$ & Pos & (37) hypo & III & OM & No & Pos & Foll $A$ with $\mathrm{OM}$ in $\mathrm{HT}$ \\
\hline 2 & 61 & $\mathrm{~F}$ & Neg & (10) hypo & II & PTC with OM & Yes & Neg & PTC with OM in MNG \\
\hline 3 & 55 & M & Neg & (60) iso & III & OM & Yes & Pos & MNG with OM \\
\hline 4 & 56 & $\mathrm{~F}$ & Neg & (22) iso & II & OM & No & Neg & $\mathrm{HC}-\mathrm{A}$ \\
\hline 5 & 50 & M & Neg & (16) iso & III & OM & No & Neg & Foll A with OM \\
\hline 6 & 44 & $\mathrm{~F}$ & Pos & (30) hypo & II & OM & Yes & Pos & Warthin's-like PTC in HT \\
\hline 7 & 23 & $\mathrm{~F}$ & Pos & (27) hypo & III & PTC with OM & Yes & Pos & Warthin's-like PTC in GD \\
\hline 8 & 45 & $\mathrm{~F}$ & Neg & (31) hypo & III & $\mathrm{HC}-\mathrm{N}$ & Yes & Pos & HC-A \\
\hline 9 & 29 & $\mathrm{~F}$ & Neg & (25) iso & III & OM & No & Neg & Foll $A$ with $\mathrm{OM}$ and occult PTC \\
\hline 10 & 69 & $\mathrm{~F}$ & Neg & (15) hypo & III & OM & Yes & Pos & Foll $\mathrm{HC}-\mathrm{C}$ in $\mathrm{HT}$ \\
\hline 12 & 42 & M & Neg & (15) hypo & II & $\mathrm{HC}-\mathrm{N}$ & Yes & Pos & Foll HC-C \\
\hline 13 & 47 & $\mathrm{~F}$ & Neg & (17) hypo & III & OM & Yes & Pos & Foll HC-C in focal HT \\
\hline 14 & 65 & $\mathrm{~F}$ & Pos & (15) hypo & II & $\mathrm{HC}-\mathrm{N}$ & Yes & Pos & PTC with OM in HT \\
\hline 15 & 53 & $\mathrm{~F}$ & Neg & (25) hypo & III & OM & Yes & Pos & Foll HC-C in HT \\
\hline 16 & 50 & $\mathrm{~F}$ & Neg & (20) hypo & III & $\mathrm{HC}-\mathrm{N}$ & No & Pos & $\mathrm{HC}-\mathrm{A}$ \\
\hline 17 & 72 & M & Neg & (11) iso & II & OM & No & Neg & Nodular HT and occult PTC \\
\hline 18 & 51 & $\mathrm{~F}$ & Neg & (27) iso & III & OM & Yes & Pos & Foll $\mathrm{HC}-\mathrm{C}$ \\
\hline 19 & 52 & $\mathrm{~F}$ & Neg & (30) iso & II & $\mathrm{HC}-\mathrm{N}$ & No & Neg & OM in MNG \\
\hline 20 & 70 & $\mathrm{~F}$ & Neg & (25) hypo & III & OM & No & Pos & Foll HC-C \\
\hline 21 & 57 & $\mathrm{~F}$ & Pos & (12) iso & II & PTC with OM & Yes & Neg & PTC with OM in HT \\
\hline 22 & 60 & $\mathrm{~F}$ & Neg & (26) hypo & III & OM & Yes & Pos & Foll $\mathrm{HC}-\mathrm{C}$ \\
\hline \multicolumn{10}{|c|}{$\begin{array}{l}M=\text { male; } F=\text { female; hypo }=\text { hypoechoic; iso }=\text { isoechoic; } N=\text { neoplasia; } C=\text { carcinoma; } A=\text { adenoma; Foll = follicular; } P T C=\text { papillary thyroid carcinoma; } H C=\text { Hürthle cells; } O M=\text { oncocytic } \\
\text { metaplasia; } G D=\text { Graves' disease; } H T=\text { Hashimoto's thyroiditis; } M N G=\text { multinodular goiter. } \\
{ }^{*} \text { CFDS pattern was classified } 0-\text { III as reported in (28). }\end{array}$} \\
\hline
\end{tabular}


HC represented $>75 \%$ of the total follicular cells present on the smear. HCT were classified as follicular unless typical nuclear features of PTC were detected within the oxyphilic cells - in this case a cytological diagnosis of papillary variant of HCT (or Warthin'slike PTC) was performed. A lower number of $\mathrm{HC}$ was considered as evidence of OM (focal: $10-20 \% \mathrm{HC}$; moderate: $21-50 \% \mathrm{HC}$; marked $51-75 \% \mathrm{HC}$ ), which was associated with various patterns of other benign, suspect or malignant cytological features (see Table 1).

Surgical specimens were fixed with $10 \%$ tamponated formalin. Each nodule was totally or subtotally sampled (with at least 10 sections including the capsule) and included in paraffin. Serial slides stained with HE were observed. The presence of benign or malignant neoplasias, as well as non-neoplastic lesions was identified by common criteria. With regard to the oxyphilic cells, the final histotype was classified as HCT when $\mathrm{HC}$ were $>75 \%$ and as $\mathrm{OM}$ (associated with various different benign or malignant thyroid lesions) when $10-75 \%$ of $\mathrm{HC}$ were detected.

\section{Statistical analysis}

The sensitivity and specificity for the correct identification of malignant nodules were calculated for the diagnostic procedures (single or in combination) by the Galen \& Gambino formula (29).

\section{Results}

\section{Cytological and histological findings}

As shown in Table 1, 16 (66.7\%) nodules were considered malignant or suspect for malignancy by FNAC criteria, while the remaining eight (33.3\%) were not suspect. Histological examination showed 14 malignant and 10 benign lesions; in two of the latter cases a small occult PTC was also detected (Table 1), but this result was not considered when the sensitivity and specificity of FNAC was calculated (see below). As shown in Table 2, the sensitivity of FNAC for malignancy was $92.8 \%$ and the specificity was $70.0 \%$.

With regard to the oncocytic changes, histological examination revealed the following: (a) 14 cases of HCT: 11 malignant (seven follicular carcinomas, three Warthin's-like PTC and one HC-PTC) and three benign $\mathrm{HC}$ adenomas; (b) 10 cases displayed $\mathrm{OM}$

Table 2 Comparison between cytological and histological findings.

\begin{tabular}{lccc}
\hline FNAC & Malignant & & Benign \\
\hline Suspect & 13 & & 3 \\
Not suspect & 1 & & 7 \\
Sensitivity & & $92.8 \%$ & \\
Specificity & & $70.0 \%$ & \\
\hline
\end{tabular}

associated with three PTC and seven benign lesions (three follicular adenomas, three nontoxic multinodular goiters and one nodular Hashimoto's thyroiditis). Two cases of occult PTC were detected in benign thyroid lesions (one follicular adenoma and one nodular Hashimoto's thyroiditis).

The ability of FNAC to differentiate true HCT from $\mathrm{OM}$ is reported in Table 3. True HCT were correctly identified by FNAC in only 5/14 nodules, providing a sensitivity of $35.7 \%$; on the other hand, FNAC correctly identified $\mathrm{OM}$ in $7 / 10$ cases, corresponding to a specificity of $70 \%$.

\section{Results of MIBI scan}

As reported in Table 4, 15/24 (62.5\%) nodules displayed early and late MIBI uptake. No significant difference in MIBI positivity was found between malignant $(10 / 15=66.7 \%)$ and benign $(5 / 9=55.5 \%)$ nodules. The ability of MIBI to distinguish true HCT from $\mathrm{OM}$ is also reported in Table 4: most $(11 / 14=78.5 \%)$ of the true HCT were MIBI-positive, while a few $(4 / 10=40 \%)$ thyroid lesions with OM displayed significant MIBI uptake. This corresponded to a sensitivity of $78.5 \%$ and a specificity of $60 \%$ in correctly identifying true HCT.

\section{Combination of FNAC and MIBI scan in the diagnostic evaluation of HC nodules}

Finally, we evaluated the potential value of a combined approach by MIBI scan and FNAC to thyroid nodules with HC cytology. As reported in Table 5, the sensitivity

Table 3 Oncocytic features: comparison between cytological and histological findings.

\begin{tabular}{lccccc}
\hline & \multicolumn{3}{c}{ Histology } \\
\cline { 2 - 5 } & \multicolumn{2}{c}{ HCT } & & \multicolumn{2}{c}{$\begin{array}{c}\text { Oncocytic } \\
\text { metaplasia }\end{array}$} \\
\cline { 2 - 3 } \cline { 5 - 6 } FNAC & 3 & 2 & & 1 & 2 \\
HCT (8) & Malignant & Benign & Malignant & Benign \\
Oncocytic metaplasia (16) & 8 & 1 & & 2 & 5 \\
\hline
\end{tabular}

Table 4 Comparison between MIBI results and malignant and benign thyroid tumors, and histological types.

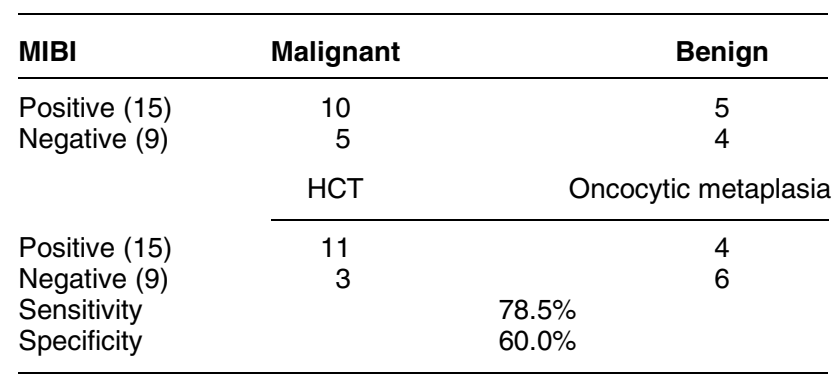


Table 5 Comparison between cytological and histological findings in MIBI-positive and -negative nodules.

\begin{tabular}{llcc}
\hline MIBI & FNAC & Malignant & Benign \\
\hline Positive (15) & Suspect & 9 & 3 \\
& Non-suspect & 1 & 2 \\
Negative (9) & Suspect & 4 & 0 \\
& Non-suspect & 0 & 5 \\
\hline
\end{tabular}

and specificity of FNAC for malignancies in the 15 MIBI-positive nodules was $90 \%$ and $40 \%$ respectively, while in the small subgroup of nine MIBI-negative nodules both sensitivity and specificity were 100\%.

\section{Discussion}

The results of the present study confirm that FNAC has a lower diagnostic accuracy in thyroid nodules when cells with oxyphilic changes are present in the smear $(1,4,5)$, mostly represented by a reduction in the specificity. The recent observation that MIBI scan is often positive in HC thyroid neoplasias (27) prompted us to ascertain whether and to what extent the addition of MIBI scan to FNAC could improve the diagnostic evaluation of 'cold' thyroid nodules with HC cytology. The results obtained did not provide any evidence that in this particular subgroup of thyroid nodules MIBI scan could improve the sensitivity and specificity in the pre-surgical detection of malignant tumors. Our data are in keeping with previous studies $(23,27)$ including a limited series of thyroid HC tumors (27).

Independently from the benign or malignant histotype, in our study the large majority $(78.5 \%)$ of true HCT displayed an MIBI-positive scan, while only a minority $(40 \%)$ of thyroid lesions with OM were MIBIpositive. This finding suggests a direct relationship between MIBI uptake and number/mass of oncocytes, which present high affinity and uptake for MIBI $(23,27)$. This phenomenon is believed to be due to crowded mitochondria (corresponding to the eosinophilic cytoplasmatic granules) independently from malignancy. It is interesting to note that in the present study, MIBI scan provided a higher sensitivity for the correct identification of true HC tumors when compared with FNAC. On the other hand, the specificity of MIBI in the identification of true HCT was slightly lower than that of FNAC. No further increase in the diagnostic accuracy for thyroid cancer was observed when MIBI scan and FNAC were combined.

In conclusion, the present study shows that most $\mathrm{HC}$ tumors are MIBI-positive, but this scan is not sufficiently specific to differentiate true HC neoplasias from other thyroid lesions showing $\mathrm{HC}$ at FNAC, although an MIBI-negative scan strongly supports the absence of true HC tumors. MIBI scintiscan has no value in differentiating malignant from benign $\mathrm{HC}$ neoplasias: due to the high proportion of malignancy observed in HC tumors, a surgical approach is generally advised.

\section{Acknowledgements}

This work was partially supported by M.U.R.S.T. (Rome, Italy) and by funds from the Regione Autonoma Sardegna to the Centro Studio per la Prevenzione e Terapia delle Malattie della Tiroide.

\section{References}

1 Gonzalez JL, Wang HH \& Ducatman BS. Fine-needle aspiration of Hürthle cell lesions. A cytomorphologic approach to diagnosis. American Journal of Clinical Pathology $1993100231-235$.

2 Grant CS. Operative and postoperative management of the patient with follicular and Hürthle cell carcinoma. Do they differ? Surgical Clinics of North America 199575 395-403.

3 Bronner M \& Li Volsi V. Oxyphilic (Askanazy/Hürthle cell) tumors of the thyroid: microscopic features predict biological behaviour. Surgical Pathology 19881 137-150.

4 Carcangiu ML, Bianchi S, Savino D, Voynick IM \& Rosai J. Follicular Hürthle cell tumors of the thyroid gland. Cancer 1991 68 1944-1953.

5 McIvor NP, Freeman JL, Rosen I \& Bedard YC. Value of fine-needle aspiration in the diagnosis of Hürthle cell neoplasms. Head and Neck $199315335-341$.

$6 \mathrm{Li}$ Volsi V. Surgical Pathology of the Thyroid, pp 272-288. Philadelphia: WB Saunders, 1990.

7 Har-El G, Hadar T, Segal K, Levy R \& Sidi J. Hürthle cell carcinoma of the thyroid gland. A tumor of moderate malignancy. Cancer $1986571613-1617$.

8 Beckner ME, Heffess CS \& Oertel JE. Oxyphilic papillary thyroid carcinomas. American Journal of Clinical Pathology 1995103 $280-287$.

9 Cheung CC, Ezzat S, Ramyar L, Freeman JL \& Asa SL. Molecular basis of Hürthle cell papillary thyroid carcinoma. Journal of Clinical Endocrinolgy and Metabolism $2000 \mathbf{8 5} 878-882$.

10 Belfiore A \& La Rosa GL. Fine-needle aspiration biopsy of the thyroid. Endocrinology and Metabolism Clinics of North America 200130 361-400.

11 Renshaw AA. Accuracy of thyroid fine-needle aspiration using receiver operator characteristic curves. American Journal of Clinical Pathology $2001116477-482$.

12 Papini E, Guglielmi R, Bianchini A, Crescenzi A, Taccogna S, Nardi F et al. Risk of malignancy in nonpalpable thyroid nodules: predictive value of ultrasound and color-Doppler features. Journal of Clinical Endocrinolgy and Metabolism 200287 1941-1946.

13 Gharib H. Changing concepts in the diagnosis and management of thyroid nodules. Endocrinology and Metabolism Clinics of North America 199726 777-800.

14 Baillet GY, Mena IG, Kuperus JH, Robertson JM \& French WJ. Simultaneous technetium-99m MIBI angiography and myocardial perfusion imaging. Journal of Nuclear Medicine 198930 38-44.

15 Iskandrian AS, Heo J, Kong B, Lyons E \& Marsch S. Use of technetium-99m isonitrile (RP-30A) in assessing left ventricular perfusion and function at rest and during exercise in coronary artery disease, and comparison with coronary arteriography and exercise thallium-201 SPECT imaging. American Journal of Cardiology $198964270-275$.

16 Kiat H, Maddahi J, Roy LT, Van Train K, Friedman J, Resser K et al. Comparison of technetium 99m methoxy isobutyl isonitrile and thallium 201 for evaluation of coronary artery disease by planar and tomographic methods. American Heart Journal 1989 $1171-11$.

17 Taillefer R, Lambert R, Dupras G, Gregoire J, Leveille J, Essiambre R et al. Clinical comparison between thallium-201 and 
Tc-99m-methoxy isobutyl isonitrile (hexamibi) myocardial perfusion imaging for detection of coronary artery disease. European Journal of Nuclear Medicine 198915 280-286.

18 Aktolun C, Bayhan H \& Kir M. Clinical experience with Tc-99m MIBI imaging in patients with malignant tumors. Preliminary results and comparison with Tl-201. Clinical Nuclear Medicine $199217171-176$

19 Geatti O, Shapiro B, Orsolon PG, Proto G, Guerra UP, Antonucci F et al. Localization of parathyroid enlargement: experience with technetium-99m methoxyisobutylisonitrile and thallium-201 scintigraphy, ultrasonography and computed tomography. European Journal of Nuclear Medicine 199421 17-22.

20 Hassan IM, Sahweil A, Constantinides C, Mahmoud A, Nair M, Omar YT et al. Uptake and kinetics of Tc-99m hexakis 2-methoxy isobutyl isonitrile in benign and malignant lesions in the lungs. Clinical Nuclear Medicine 198914 333-340.

21 Taillefer R, Boucher Y, Potvin C \& Lambert R. Detection and localization of parathyroid adenomas in patients with hyperparathyroidism using a single radionuclide imaging procedure with technetium-99m-sestamibi (double-phase study). Journal of Nuclear Medicine 199233 1801-1807.

22 Balon HR, Fink-Bennet TD \& Stoffer SS. Technetium-99m-sestamibi uptake by recurrent Hürthle cell carcinoma of the thyroid. Journal of Nuclear Medicine 199233 1393-1395.

23 Foldes I, Levay A \& Stotz G. Comparative scanning of thyroid nodules with technetium-99m pertechnetate and technetium99m methoxyisobutylisonitrile. European Journal of Nuclear Medicine 199320 330-333.

24 Nakahara H, Noguchi S, Murakami N, Hoshi H, Jinnouchi S, Nagamachi $\mathrm{S}$ et al. Technetium-99m-sestamibi scintigraphy compared with thallium-201 in evaluation of thyroid tumors. Journal of Nuclear Medicine 199637 901-904.

25 Scott AM, Kostakoglu L, O’Brien JP, Straus DJ, Abdel-Dayem HM \& Larson SM. Comparison of technetium-99m-MIBI and thallium201-chloride uptake in primary thyroid lymphoma. Journal of Nuclear Medicine 199233 1396-1398.

26 Vattimo A, Bertelli P \& Burroni L. Effective visualization of suppressed thyroid tissue by means of baseline $99 \mathrm{mTc}-\mathrm{methoxy}$ isobutyl isonitrile in comparison with $99 \mathrm{mTc}$-pertechnetate scintigraphy after TSH stimulation. Journal of Nuclear Biology Medicine 199236 315-318.

27 Vattimo A, Bertelli P, Cintorino M, Burroni L, Volterrani D \& Vella A. Identification of Hürthle cell tumor by single-injection, double-phase scintigraphy with technetium-99m-sestamibi. Journal of Nuclear Medicine $199536778-782$.

28 Bogazzi F, Bartalena L, Brogioni S, Burelli A, Manetti L, Tanda MI et al. Thyroid vascularity and blood flow are not dependent on serum thyroid hormone levels: studies in vivo by color flow Doppler sonography. European Journal of Endocrinology 1999 $140452-456$.

29 Galen RS \& Gambino RS. In How to determine the predictive value and efficiency of a test when reading a scientific paper. Beyond normality: The predictive value and efficiency of medical diagnoses, pp 29-40. New York: John Wiley \& Sons, 1975.

Received 4 June 2003

Accepted 2 September 2003 